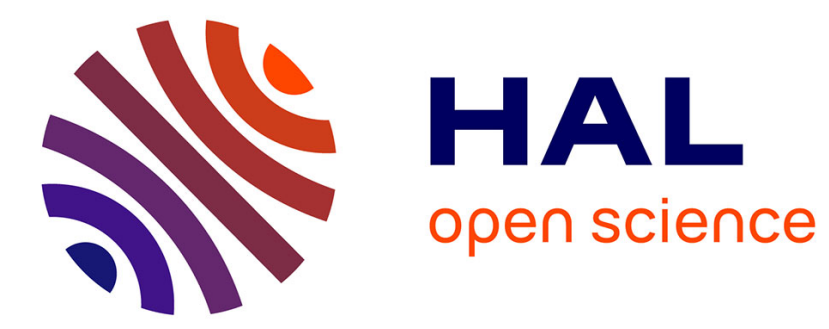

\title{
Une nouvelle mosaïque trouvée à Lyon
}

Henri Stern

\section{To cite this version:}

Henri Stern. Une nouvelle mosaïque trouvée à Lyon. Gallia - Fouilles et monuments archéologiques en France métropolitaine, 1975, 33 (1), pp.159-170. 10.3406/galia.1975.1517 . hal-01937293

\section{HAL Id: hal-01937293 https://hal.science/hal-01937293}

Submitted on 11 Mar 2020

HAL is a multi-disciplinary open access archive for the deposit and dissemination of scientific research documents, whether they are published or not. The documents may come from teaching and research institutions in France or abroad, or from public or private research centers.
L'archive ouverte pluridisciplinaire HAL, est destinée au dépôt et à la diffusion de documents scientifiques de niveau recherche, publiés ou non, émanant des établissements d'enseignement et de recherche français ou étrangers, des laboratoires publics ou privés.

\section{(ㅇ)(1) $\$$}

Distributed under a Creative Commons Attribution - NonCommercial - NoDerivatives| 4.0 


\title{
UNE NOUVELLE MOSÄ̈QUE TROUVÉE A LYON*
}

\author{
par Henri STERN
}

En décembre 1967, lors de travaux de terrassement pour l'installation d'une grue nécessaire à la percée du tunnel de l'autoroute, une mosäque romaine a été trouvée au pied de la Montée de Choulans à Lyon. En raison des travaux en cours, l'enlèvement a été fait avec la plus grande rapidité.

La mosaïque a été découverte dans un quartier de Lyon où, jusqu'alors, aucune trouvaille de ce genre n'avait été signalée (elle est située à $350 \mathrm{~m}$ au sud du point le plus méridional où des pavements romains ont été trouvés sur la rive droite de la Saône) ${ }^{1}$. Le pavement était orienté du sud au nord avec une légère déviation $\left(15^{\circ}\right)$ vers le n.-o. (fig. 1). Voici les mesures que donne M. Bassier : longueur (du nord au sud, comprenant la rallonge au rinceau de calices et de cœurs) et allant jusqu'à la limite de la fouille au nord, $3,75 \mathrm{~m}$; largeur de toute la partie fouillée y comprise la bande de raccord blanche à l'est, $2,75 \mathrm{~m}$. En réalité la mosaïque devait mesurer au moins $4 \mathrm{~m}$ du nord au sud et $3 \mathrm{~m}$ d'est en ouest.

La rallonge au sud qui ne s'étend que sur la largeur du tapis sans bordure est comme un seuil, évidemment trop large pour une simple porte $(2,50 \mathrm{~m})$. Peut-être s'agissait-il d'une entrée dépourvue de vantaux qui donnait sur une cour intérieure. Quoi qu'il en soit, l'accès de la pièce a dû se trouver de ce côté, le tableau central étant orienté de façon à être vu du sur.

Le pavement était largement mutilé au moment du dégagement (fig. 1). Toute la partie nord-ouest sur une ligne allant de l'angle nord-est vers l'angle sud-ouest était détruite, mais la partie centrale était heureusement préservée. L'angle sud-est a également disparu dans une large brèche qui s'étendait jusque sous le tableau central pour rejoindre ensuite la brèche nord-ouest.

- Tous les rerseignements malériels donnes, ci-apres, sur celte mosaique sont dus a l'amabilite de M. Cl. Bascier, ingénieur-conseil à Périgueux, qui avait été chargé de lenlevement. Le pavement se trouve actuellement entoilé à latelier de .I. Bassier qui attend d'êtr: chargé du remontage. Les clichés des fig. 1, 2, 4, 5, 6 et 7 sont de .11. Cl. Bassier, celui de la fig. $x$ provient du Vusé Saint-Pierre a Vienne. Les dessins fig. 3 et 9 sont de M. R. Prudhomme.

l if. II. Strkx, Recueil général des mosaíques de la liaule, Xe suppl. à riallia, II par la suite= Recueil, n" 31, et le plan de L youn à la fin du volume. 


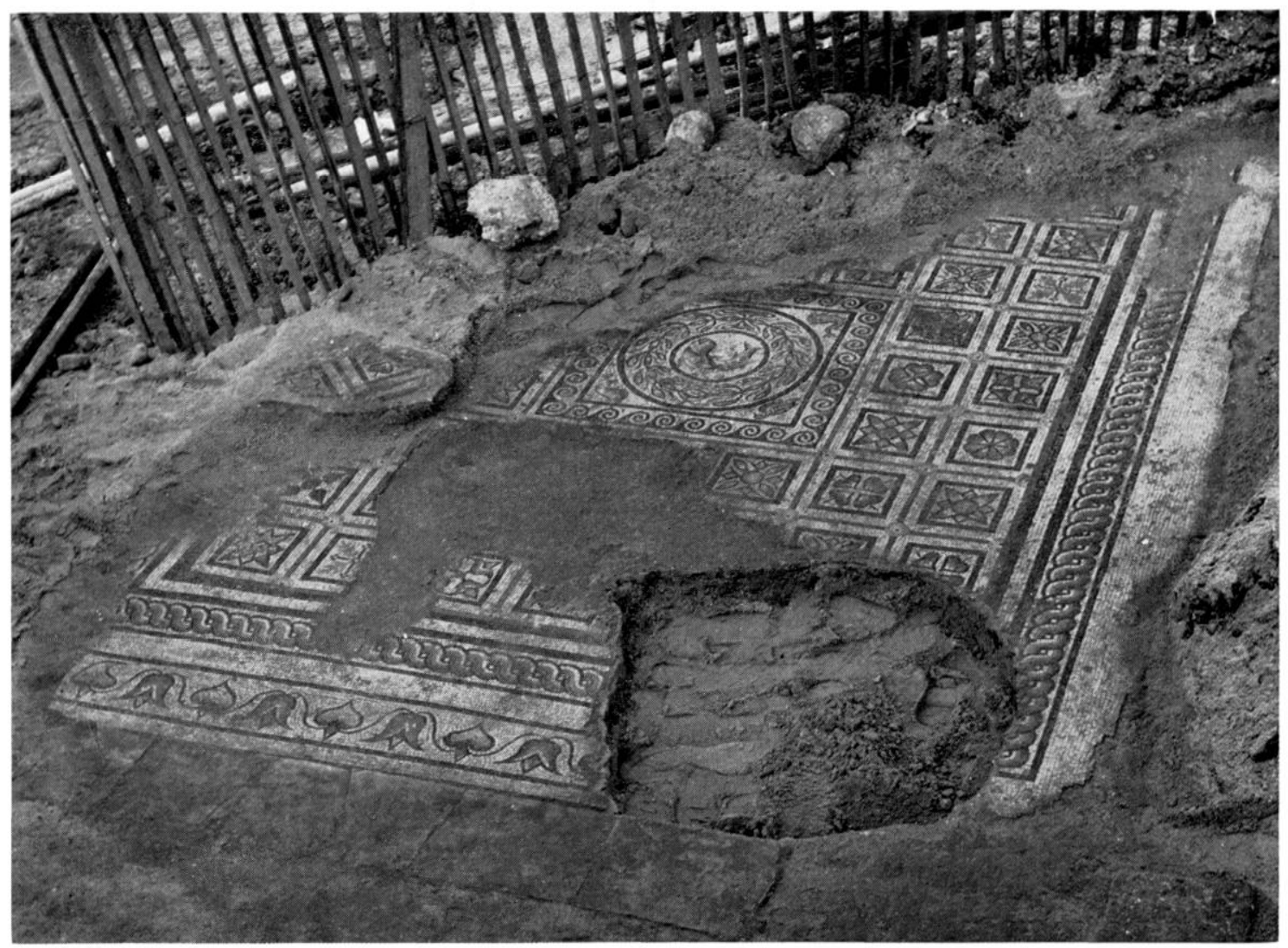

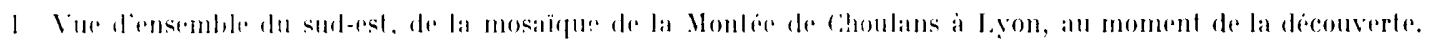

Le tapis proprement dit d'environ 2,50 m sur 3 m est couvert d'un quadrillage de $7 \times 9$ !'asés dont !es 9 du milieu sont remplacées par un tableaul carré entouré d'une bordure de postes noir sur blane (fig. 1). Ce carré inscrit un médaillon circulaire entouré d'une couronne de lauriers entre deux filets doubles noirs et, en son milies, un poisson dont le mouvement fretillant (fig. i) épouse le tracé circulaire du cadre. Dans les quatre écoincons se placent une paire de canards ef une autre de dauphins qui, chacune, se font face sur les diagonales.

Les cases du quadrillage sont garnies de carrés remplis de motifs ornementaux dont on partera plus loin. Ce tapis est bordé (en allant de l'intérieur à l'extérieur) d'un filet double rouge, d'une bande noire t trois rangs de (ables), d'une autre blanche (quatre rangs de cubes), d'une troisiome noire (trois ranges de cubes). d'un entrelaces à deux brins, faits rharun de trois filets simples, rouge, jaune et blanc, et d'un contour noir (fig. 2). Line bande de raceord blanche qui comporte jusqu'à dix-sept rangs rle cubes parallèles au cardre du tapis, n'est visible que sur le còtre est.

Sur le còte sud, la rallonge ou plutòt le seuil (fig. 1 (at 2) n'est que partiellement conservé. On en voit le point d'arrêt vers l'angle sud-ouest oì le filet qui le borde retourne à angle droit, exactement à l'aplomb du filet noir qui accompagne l'entrelares à l'intérieur (fig. 2 , a gauche). A l'extrémiti opposée, la mosaïque est détériorée, mais un morceau du fond hanc à l'extérieur du lapis rot conserve /fige. 1, à droite). Il comporte treize rangs de cubes 


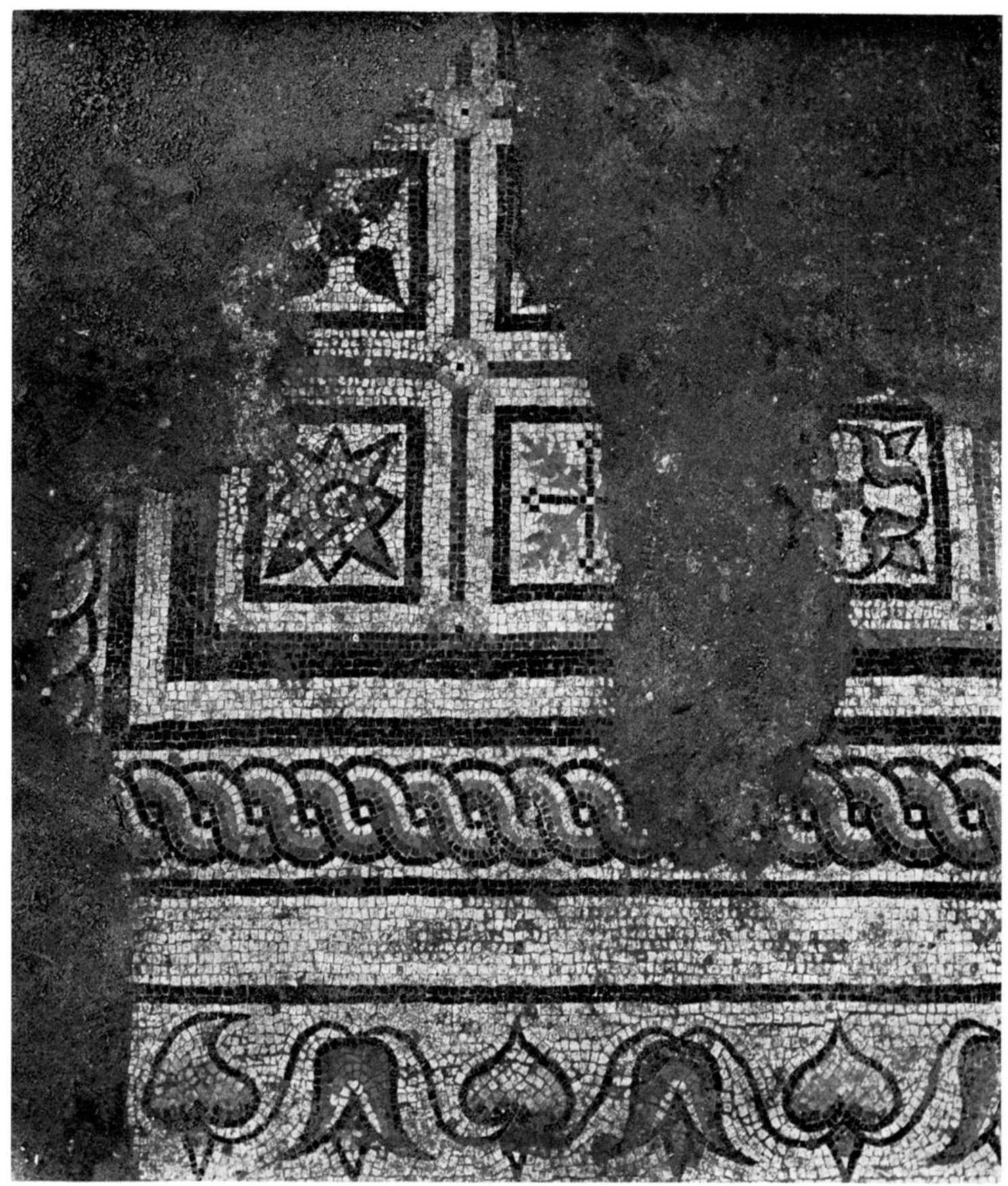

$\because$ Parlie sud-outest de la mosaïrue, on place.

blanes paralledes alu dessin du seuil. alors que crelui-ri n'est distant du tapis que de huit rangs. Il se terminait donc au mème point à l'angle sud-est qu'à l'angle opposí. Il forme un rectangle allongé dessinc pare un filet double noir et garni d'une bande de calieses de de cours alternés tète-berche. reliés entre rux par des tiges en s allongés. A l'extremite ouest, la feuille cordiforme se transforme en une sorte de feuille le lierre recourbere qui, comme une parenthese, arrete le rinceatu. Les contours sont noirs, les caliees a fond rouge, ont une 


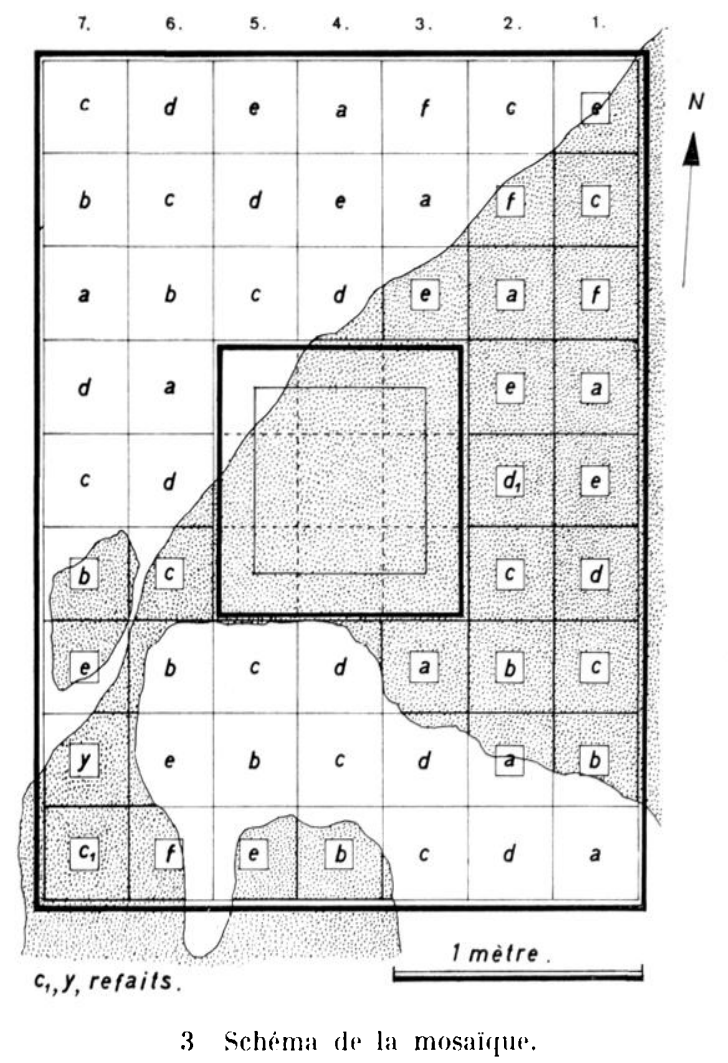

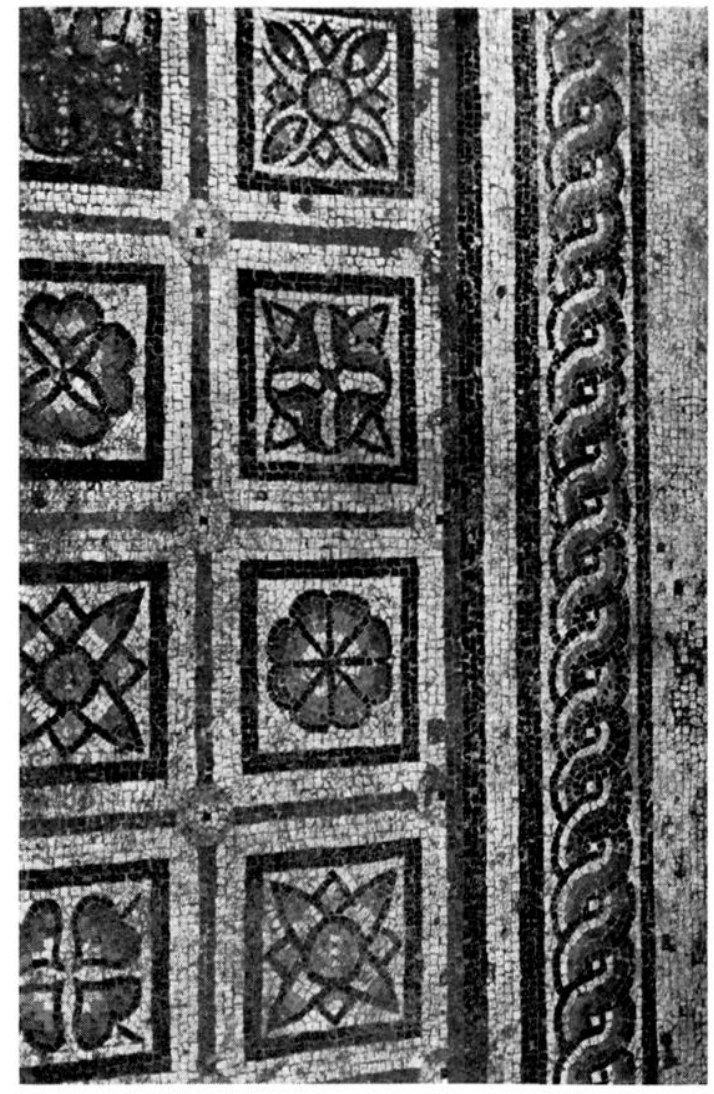

4 Cases c, d, e, a de la premiere rangere clases b, c, d, e, de la deuxicmo mangés.

bande médiane jaune, et sont blancs en haut, le pistil est gris foncé et gris clair. Les coeurs sont rouges à la partie inférieure, puis gris foncé et gris clair ; le triangle sous le cour est jaune, rouge et blanc ${ }^{2}$.

Le quadrillage du tapis est formé par des filets rouges dont les points d'intersection sont frappés de têtes de clous gris foncé, gris clair et blanches, avec un cube noir au milieu. Le fond du tapis est blanc crème. Dans chaque case se place un carré dessiné par un filet double noir qui inscrit un motif végétal rayonnant. Sur les cinquante-quatre cases qui ont dû figurer dans ce pavement, treize sont intactes, onze partiellement conscrvécs, trente sont perdues (fig. 3). Parmi les motifs de remplissage subsistants, je n'ai relevé que six types différents ${ }^{3}$. La séquence des motifs dans la rangée longitullinale 2 , qui commence en bas par la deuxième case, la première étant détruite, est décalée d'un motif par rapport à la rangée no 1 (fig. 3). Il semble en être de même diıns la rangée no 3 par rapport à la rangée précédente, s'il est permis de conclure d'après les deux seuls motifs conservés, a et e. En admettant que ce décalage se soit poursuivi progressivement jusqu'à la rangée

2 Toutes les indicalions de couleurs sont dues a 11 . C.1. Bassier.

3 Pour la commodite de l'exposé, je numerole les rangés longitudinales de rarres de 1 a 7 en allanl de droile a gauche el je désigne les motifs de remplissage par les lettres a a f cef schema, figr. 3 , ell complaml de bas en haut. 


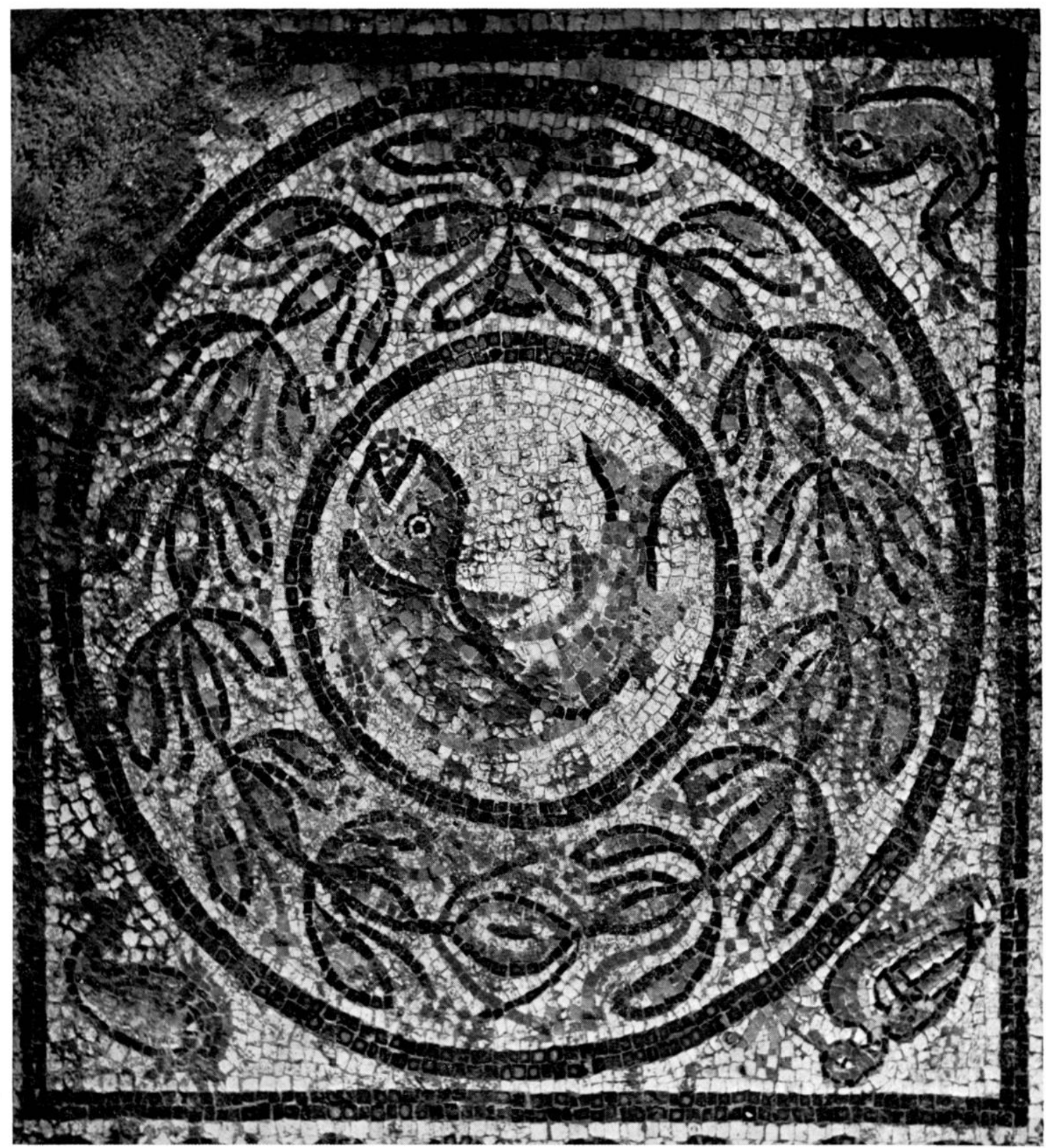

5. Medaillon central.

$n^{0} 7$, re qui semble ètre confirmé par la presence de b dans la premiere rase de la rangée

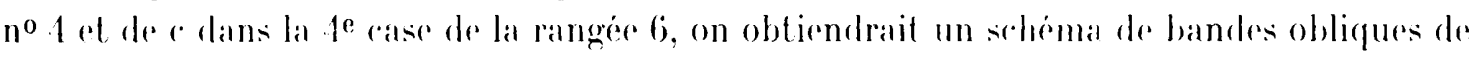

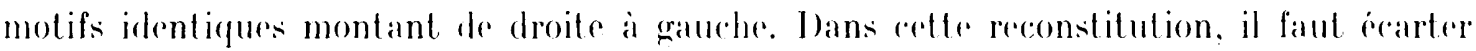
les deux motifs en bas a gauche, qui ont etr refaits dans l'antiquite at qui ne font pas partie du répertoire primitif (figr. 2).

Les motifs eux-mèmes appartiennent an répertoire ornemental rhodanien courant figr. 1. Molif a : d'un disque rayonnent quat re pelits calices a pertales filiformes el quat re pélales biconvexes, b : quatre feuilles cordiformes les liges tournées vers l'extérieur. point ent vers le centre. a : quatre pétales lancéoles al quatre pétales triangulaires en rroix rayonnent autour d'un bouton 
circulaire, -- d : fleuron à huit pétales arrondis, -- $\mathbf{d}^{1}:$ est une variante de $d$ : fleur à quatre pétales cordiformes en croix, - e : quatre calices de fleurs en croix. - $f:$ croix latine pattée avec quatre feuilles à six lobes entre les bras (fig. 2). La gamme des couleurs est celle déjà décrite : contour noir, surfaces rouges, jaunes, gris foncé, gris clair et blanches. Les feuilles autour des croix pattées sont entierement jaunes. Les cubes mesurent $1 \mathrm{~cm}^{2}$ en moyenne.

C'est dans le tableau du milieu que le mosaisste a fait de son mieux. Le travail est plus soigné, les dimensions des cubes descendent jusqu'à $0.4 \mathrm{~cm}^{2}$ et sont d'une gramme plus variće. La couronne de laurier (fig. 5) se compose de deux branches de longueur égale partant d'un point en haut sur l'axe vertical qui se rejoignent en bas dans une sorte de fermoir hiconvexe. Chaque branche est composée de six gerbes, de trois feuilles chacune, la succession des feuilles du milieu formant la branche. Deux étamines ou pistils it tige ondoyante noire flanquent la feuille centrale; ils se terminent par trois cubes rouges disposés en croix autour d'un cube blanc. Le contour des feuilles est noir, la surface est alternativement rouge et jaune ou gris clair et gris foncé, le fond est blanc crème. Le disque central est garni de l'image d'un poisson dont le corps se recourbe en un mouvement violent pour s'adapter à la circonférence du médaillon. La gueule entrouverte de l'animal laisse apparaître ses "dents", faites de cubes blancs triangulaires séparées par des cubes noirs de forme irrégulière. Pour rendre la plasticité du corps, le mosaïste a tracé la raie dorsale noire qui continue le contour de la tête en une courbe hardie vers l'extrémité raudale bifide. L'ơil rond à la pupille noire, exécuté avec beaucoup de soin, est composé de cubes d'à peine 2 ou $3 \mathrm{~mm}^{4}$.

Le cercle est cantonné de deux canards et de deux dauphins, chaque paire se faisant face sur les diagonales. Le canard, seulement de l'angle sud-est, est conservé (fig. 6) ; il picore une touffe à cinq brins, surmontée d'une fleur en croisette comme celle des étamines de la couronne. Le bec est jaune, la tête comprend des tessères vertes (en verre), le ventre est rouge, les ailes sont gris foncé, wris clair, blanches et noires. Le corps dodu du volatile, son cou mince et sa tète arrondie pris sur le vif, sont bien rendus.

Les dauphins (fig. 7) ont la gueule et la nageoire caudale rouge, le corps gris foncé, gris clair et blanc, contourné de noir. Par la courbe serrée de la queue, ils épousent exactement la forme de l'écoinçon.

"Bien que l'analyse pétrographique des tessères n'ait pas été faite avec précision, on peut affirmer que tous les cubes sont en calcaire ou en marbre. Les gris foncé, gris clair et verts sont en marbre. le rouge est un calcaire marméréen à grain trés fin, le jaune est un calcaire de type barthonien ou barjocien; le blanc est identique aux blanes que nous trouvons en général dans la plupart des mosaïques lyonnaises ou viennoises. Ia laille des tessères est asse\% irrégulière, les joints sont larges et irrégulitrs. La technique de pose ne semble pas particulièrement soignée. La surface ne paraît pas avoir été polie ou bien elle a été altérée après le polissage... L'envers est recouvert d'une mince couche de morlier rose trìs dur que nous n'avons pas pu enlever" (Cl. Bassier, lettre du 3 janvier 1972 ).

Cette mosaïque est un produit typique des ateliers rhodaniens. Le fond quadrille se retrowe sur sept pavements de Lyon ${ }^{5}$, il est fréquent dans la région de Vienne et plus au sud à Nìmes?. Le quadrillage s'y dessine le plus souvent par des bandes décoratives. Sur deux pavements seulement, l'un de Lyon ${ }^{8}$, l'autre d'Orange', il est formé par des

4 Le Lableau etant entoile el des pholographies de couleurs niayant pas de prises, M. Bassiel n'a pu me domner que de memoire quelques indications sur le's couleurs.

5 Recueil $11, n^{\circ s} 1,53,5 \%, 59,122,137$.

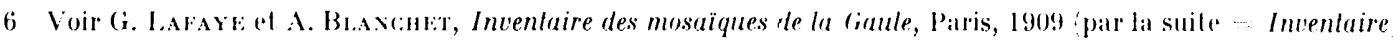
110s 15x, 173, 174, 19\%, :200, 246.

7 Inventaire, $\mathrm{n}^{\circ \mathrm{s}} \cdot 297,3 \% 9$.

$*$ Recueil 11, $n^{\circ} 137$.

9 Inventaire, $n^{\circ} 113$. 


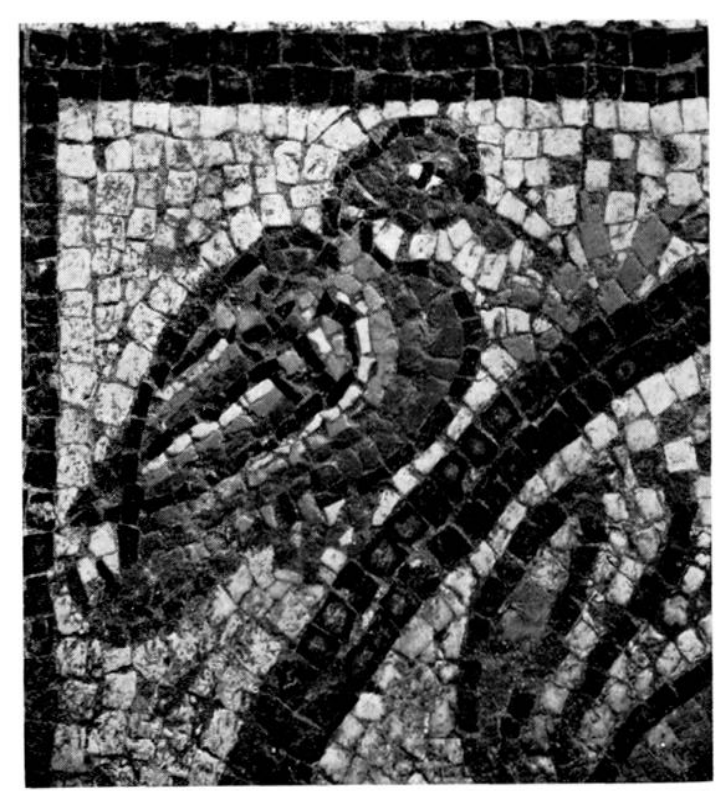

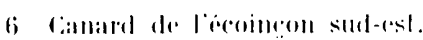

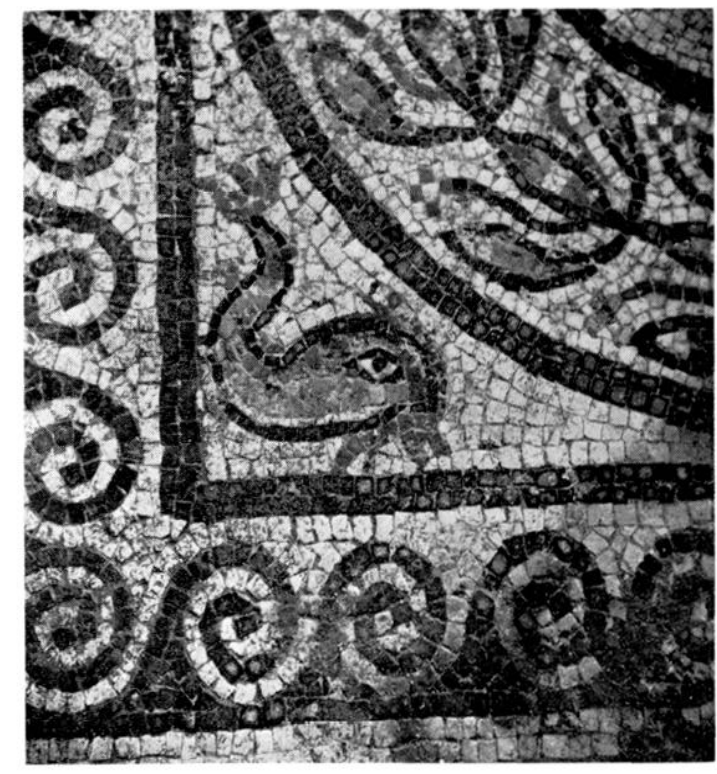

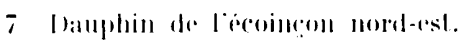

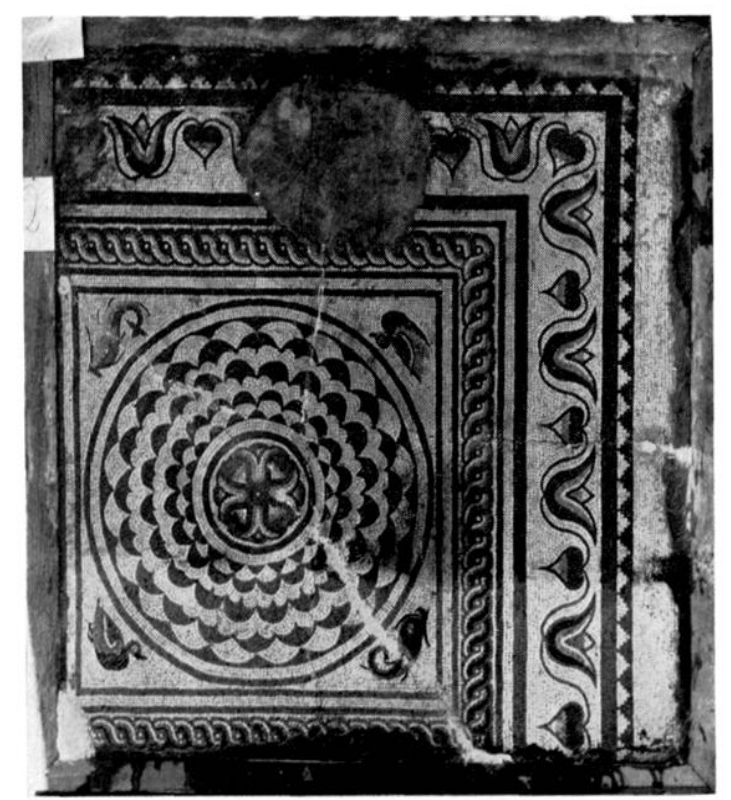

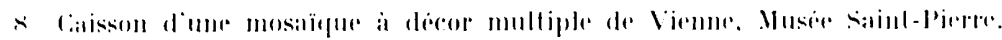

filets doubles, mosaïques qui sont assez éloignées l'une de l'autre dans le lemps (Orangre,

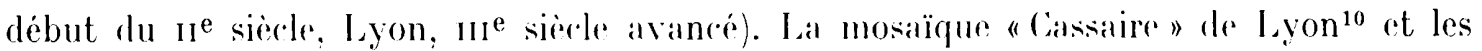
$n^{0 s}$ de l'Inerenlaire: 198 de Sainte-tolombe of 297 de Nimes, comprennent un tableau central qui occupe quatre ou neuf rases du ranevas du fond. comme la nòtre. Par le décor, la seule mosil̈que "Cassiare" se compare a celle de la .lonté de Choulans. Ses trente-six 
cases sont garnies de rosaces stylisées qui appartiennent à la même lignée mais qui sont mieux dessinés et plus complexes. Je n'ai rencontré nulle part ailleurs en Gaule les têtes de clous sur les points d'intersection du quadrillage.

Les motifs de remplissage reviennent dans des pavements rhodaniens et plus particulièrement à Lyon : a revient trois fois à Lyon ${ }^{11}$ avec cependant une différence caractéristique : sur la nouvelle mosaïque de lyon, le bouton du calice est triangulaire, les deux pétales sont des traits noirs filiformes, alors que dans les pavements cités de Lyon, les petales ${ }^{12}$ ont une surface plus large, colorée, la rourbe en est plus elégante; $b$ n'est connu que d'un seul parement lyonnaisis où il figure deux fois. Mais les mosaïstes de la ville s'en servent fréquemment dans des rombinaisons plus savantesit $; c$ se rencontre semblable, trois fois a lyon ${ }^{15}$ et ailleurs en Gaule ${ }^{16}$ d ne revient qu'une fois à Lyyon où il est plus complexe el frappé d'une croix latine' ${ }^{17}$. Parfois il se rapproche d'un assemblage de quatre feuilles cordiformes, par exemple à Lyon dans une mosaïque publiée par Artaud ${ }^{18}$, dans une autre de Vienne ${ }^{19}$, dans une troisième d'Aps ${ }^{20}$ et ailleurs; $e$ revient plusieurs fois dans la mosäque Fiacheron de Lyon ${ }^{21}$ et dans la bordure de la mosaïque d'Achille chez Lycomede de Vienner22, mais arec la différence que le cour n'est pas matérialisé. A Vëenne et ailleurs, le motif s'enrichit d'éléments intercalaires ${ }^{23}$. Le motif $f$ ne s'est pas rencontré dans la région.

Le rinceau du seuil n'est pas moins rhorlanien. L'exemple le plus proche du nòtre est la bordure d'une mosiäque à décor multiple de Vienne, dont les fragments sont entassés sous le porche de l'église Saint-Pierre (fig. 8) 24. Lne seule différence avec notre bordure est a noter : les corurs sont dépourvus de supports triangulaires. Dans une mosaïque lyonnaise ${ }^{25}$, feuilles cordiformes et ralices sont uniformément rouges, les filets ondoyants sont doublés par des ares qui les transforment en peltes, le tout est d'un aspect plus lourd et plus géométrique. Siur la mosaïque d'Endymion, de Xîmes ${ }^{26}$, les filets sont pourvus de pelites

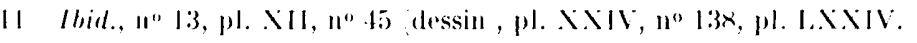

1:2 thill.

13 Itial., 1" 1:2x, 11, 1:2, pl. I.X.XI, 11, 1:2.

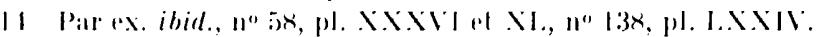

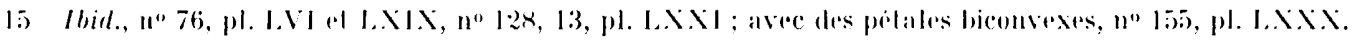

16 Par ex. Recueil I, no $1 \times 6$ a, pl. XXVI, de Dekirch, L.uximbourg.

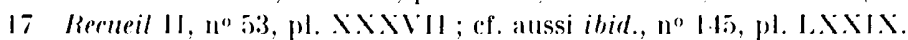

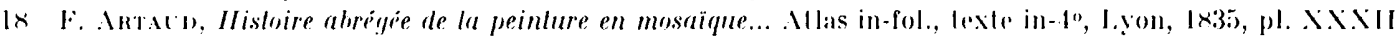

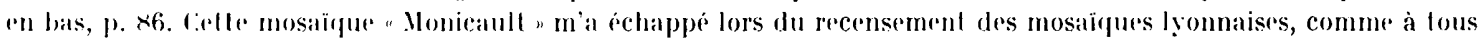
mes prederesseurs. lomission est due à une erreur de G. Lafaye, qui avail enregistre ce pavement an n 165 de Vienne.

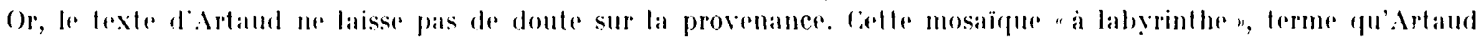

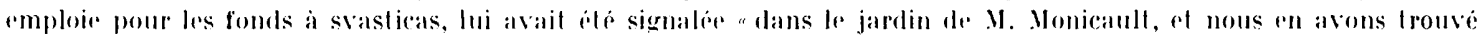
lat suile dans le poulailler du l)e Gilibert de l. yon....

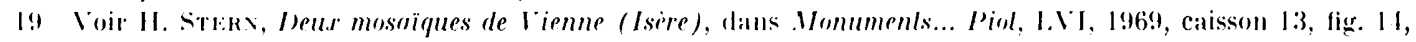
p. 31. Incenlaire, n"162, ArTato, "1.l. pl. XXl.

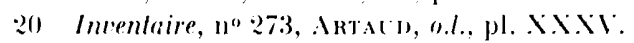

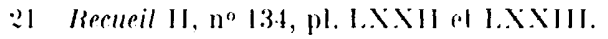

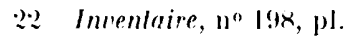

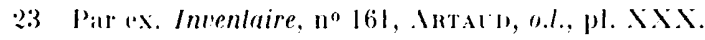

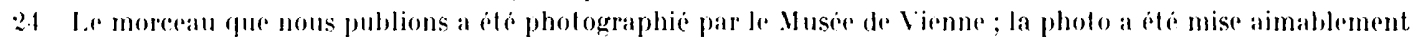

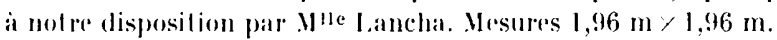

2:) Rerueil II. $1 " \times \bar{t}$, pl. I.IX.

:6 Imenlaire, 11" 330. 
pousses latérales et les calices sont bariolés de bandes de couleurs. En Suisse, où l'influence rhodanienne est prédominante ${ }^{27}$, on rencontre ce type de rinceau deux fois ${ }^{28}$, mais il est plus compliqué et assez maladroitement dessiné.

Le tableau central. I a hordure de postes (fig. 1), tout en étant rare dans la région lyonnaise ${ }^{29}$, est un motif extrêmement fréquent de la mosaïque romaine. Il en est de même de la couronne de lauriers, dont un seul échantillon a pu ètre relevé dans les parties de la Gaule recensées jusqu'à ce jour ${ }^{30}$, datant de la première moitie du rve siècle (fig. !) c'). If me von Gonzenbach en a fait connaitre trois de Suisse, tous les trois d'Avenches ${ }^{31}$, dont le second est du même type que celui de Blanzy-lès-Fismes (fig. $9 b^{\prime}$ ) : trois feuilles forment une gerbe, deux autres s'intercalent un peu plus haut de part et d'autre de la feuille centrale; la guirlande est serrée et touffue.

Le même type, plus ou moins stylisé, est fréquent en Afrique du Nord, par exemple à Sousse ('Tunisie) (fig. $9 \mathrm{~d}^{\prime}$ et $\left.\mathrm{e}^{\prime}\right)^{32}$ ou à el-I)jem (fig. $9 \mathrm{~g}^{\prime}$ et $\left.\mathrm{h}^{\prime}\right)^{33}$, qui datent de la même époque approximativement que les exemples suisses.

La guirlande de lauriers de la nouvelle mosaïque de Lyon (fig. 9 a) est d'une structure différente, nettement plus aérée : les trois feuilles des gerbes s'attachent à une petite tige commune, chaque gerbe étant agrémentée d'une paire d'étamines, mais dépourvue de feuilles intercalaires. C'est un type plus classique que celui de la Gaule et de la Suisse, et dont l'un des plus anciens exemples, de la Casa dei Gladialori, à Pompéi (fig. 9 b), remonte à la deuxième moitié du $\mathrm{I}^{\mathrm{er}}$ siècle avant notre ère ${ }^{34}$. I'autres (fig. $\left.9 \mathrm{c}\right)^{35}$ sont du II $^{\mathrm{e}}$ siècle. Un groupe nombreux qui appartient à la première moitié du siècle suivant, a été trouvé à Antioche-sur-l'Oronte (fig. 9 d, e, f, g, h) ${ }^{36}$. En l'absence d'une étude plus approfondie du sujet, ces quelques exemples, choisis un peu au hasard, peuvent suffire pour montrer que la guirlande de lauriers de notre mosaïque lyonnaise appartient à une série typologique différente, plus classique que celles de Blanzy-lès-Fismes, de la Suisse et de l'Afrique du Nord.

Les sujels figurés (fig. i). Je ne connais pas d'autres mosaïques dont le centre soit occupé par un poisson isolé. Dans un site de la province de Belgique qui n'est pas très éloigné de Lyon, à Jonvelle (Haute-Saône), on a dégagé récemment un pavement dont le tapis est décoré au milieu d'un disque garni en son centre de quatre poissons ${ }^{37}$. Dans

27 Voir V. vox Goxzkxвacr, Die römischen Mosälen der Schweiz, Bàle, 1961, passim.

28 Ibid., pl. X, 57, Hölstein, et pl. II, 29, Kloten.

29 Recueil II, no 137 , en offre le seul exemple de la ville.

30 A Blanzy-lis-Fismes, Recueil I, no 77 a et notre tig. 9 c'.

31 O.l., pl. XI.V, 388 (fig. $9 a^{\prime} ;$ et pl. LXXVII fig. $9 b^{\prime}$, , pl. V laurier-cerise .

32 (if. L. Fotcule, Inventaire des mosaiques de Sousse, Tunis, 1960, nos 57, (051 $14.57,119$.

33 In., Fouilles archéologiques de Thysdrus en 1961, Tunis, s.d., pl. XXIV a. - In., Fouilles archéologiques à Thystrus en 1960, Tunis, s.d., pl. XVI c ; les fig. 9f' et i' montrent une version simplifié du même lype de laurier.

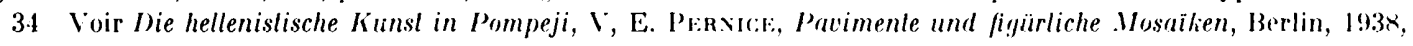
pl. X.IV, 3 ol p. 60 ot s.

35 Ie Genazano, Rome, Ilusce des Thermes.

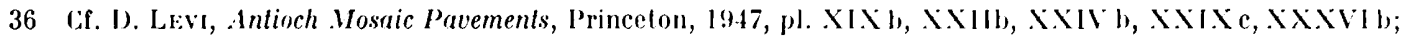
p. $388,392,393,395,397 / 8$.

37 H. Ster., Découverle d'une villa à mosaïques à Jonvelle (IIaule-taine) dans Rev. arch. de l'Esl el du CenlrpEst, X., 1969, p. 276 et s. 

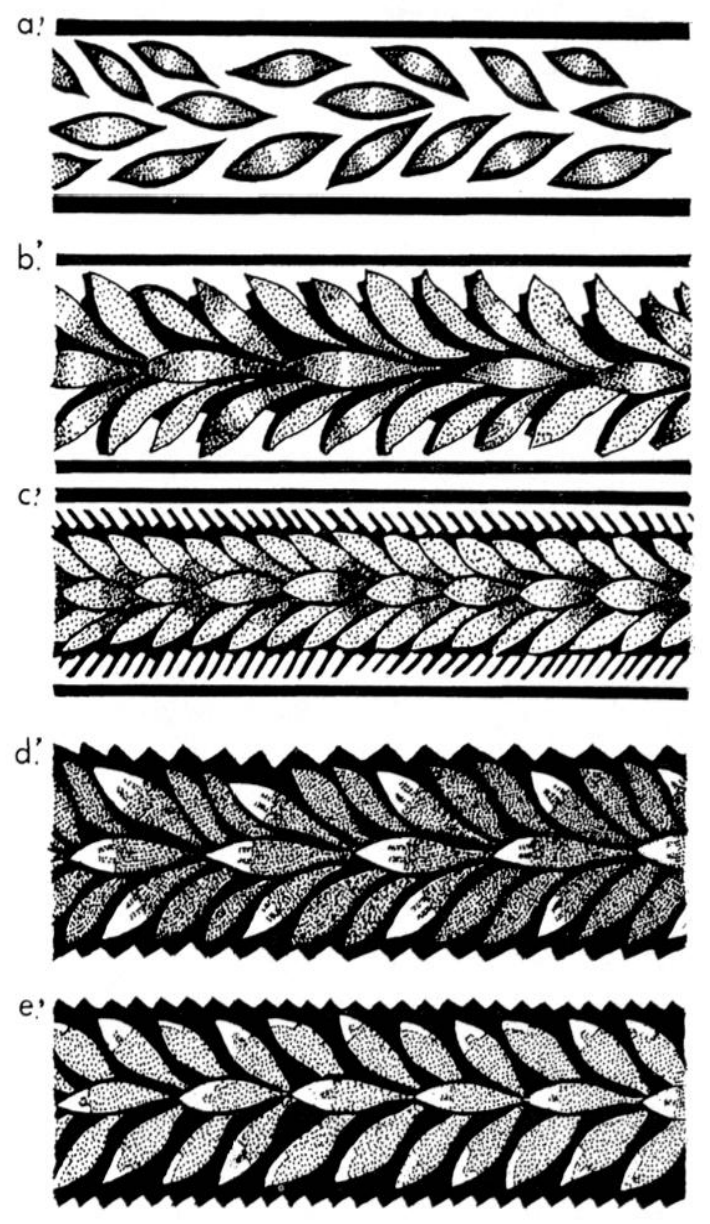

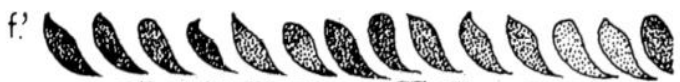

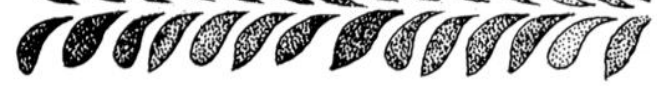
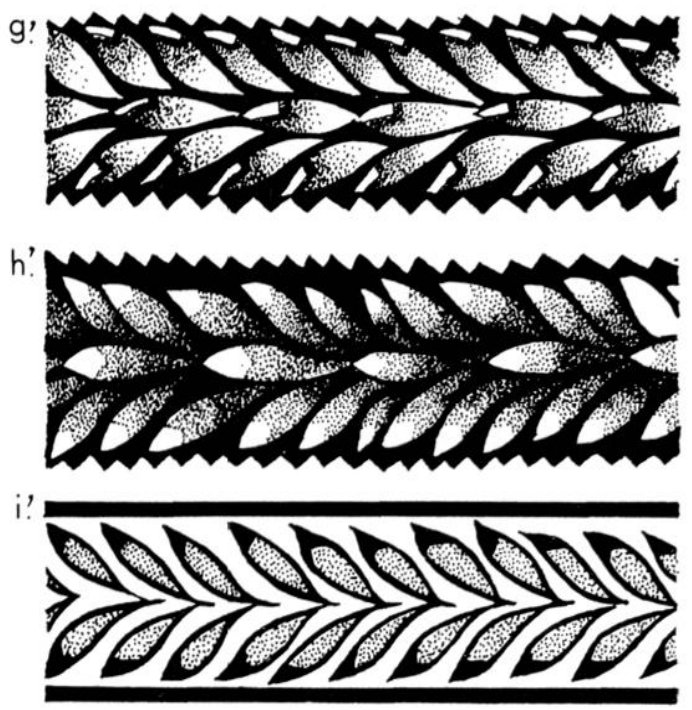
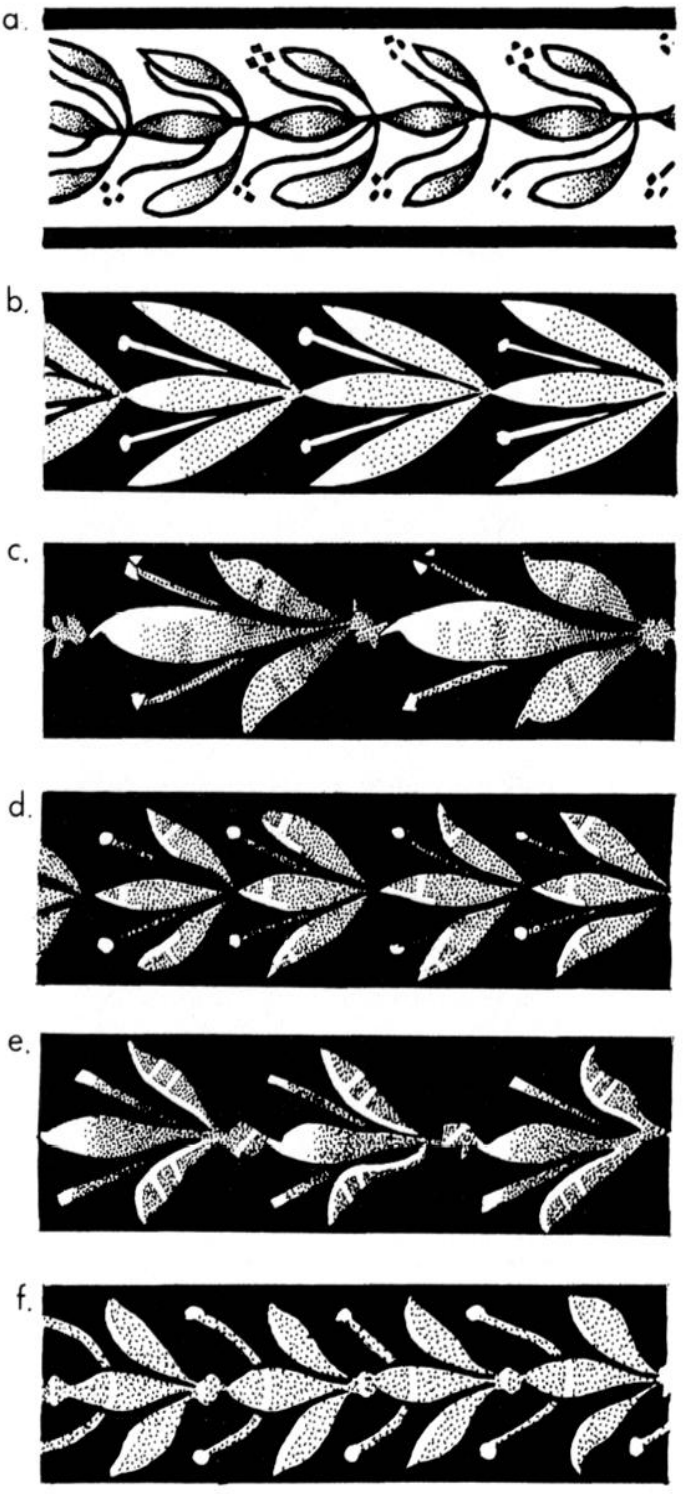

g.
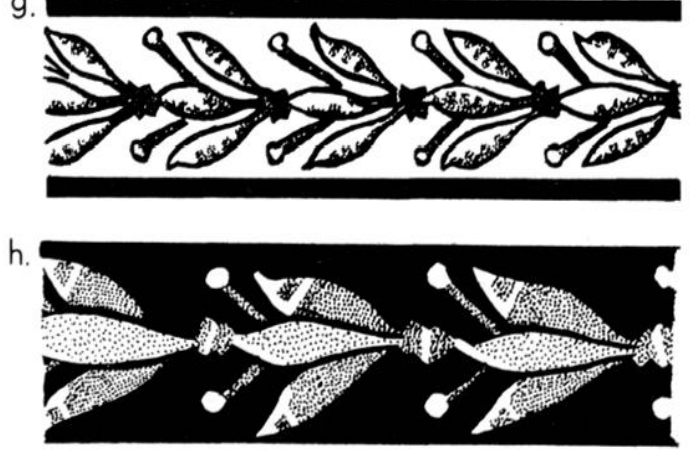

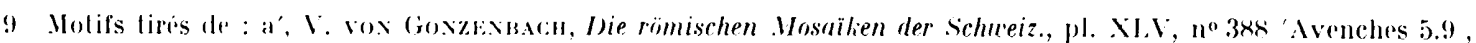

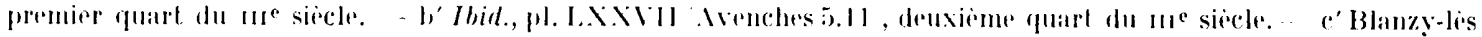


un caisson du pavement de l'leresse d'Hercule de Vienne. six poissons s'ébattent dans l'eau, encadrés d'un rinceau a rosaces stylisées, fort semblables a colles de la mosaïque lyonnaise ${ }^{38}$. Sur un seul pavement (un seuil), provenant de Sousse ${ }^{39}$, un poisson isolé est enfermé dans un médaillon; il s'enlève sur un fond clair caractérisé comme de l'eau par des traits horizontaux zigzagants. Ni par l'allure ni par sa place dans la composition il ne se compare au nòtre.

Les innombrables représentations de poissons des mosaïques africaines, italiennes, et des autres provinces de l'empire sont en général plus stéréotypées, l'animal dessiné de profil, nageant droit devant lui. Les deux mosaüques à poissons de Lyon" ne font pas exception à cette règle. Le soin avec lequel l'animal est dessiné sur la mosäque de la Nontée de Choulans laisse deviner un intérèt particulier du propriétaire pour cette image. Peut-être était-il patron de pècheries dans la Saône toute proche.

La fonction décorative que remplissent les canards et les dauphins ici en garnissant les écoinçons leur est couramment impartie dans les mosäques rhodaniennes. Sur le pavement. à décor multiple de Vienne, cité plus haut (fig. 8), ils occupent exactement less mèmes emplacements, mais au lieu de s'affronter par paires, ils se suivent dans le sens contraire à la marche d'une aiguille de montre. Le style est plus banal, mais d'un plus grand savoirfaire. Et voici quelques autres exemples rhodaniens de volatiles et de poissons remplissant des écoincons : Lyon, Recueil II, no 53, caisson met ce, pl. XXXIII, XXXV. XXXVII et XXXV'III ; Vienne, Ivresse d'Hercule, caissons $6,8,21,28^{41}$; Invenlaire, no 200 , ('aissons 9 et 13 ; Nîmes, Inventaire, no $1677^{42}$.

Dans la mosaïque de la Déserte, de Lyon ${ }^{43}$, l'exécution des dauphins est plus fine; dans le pavement Cucherat ${ }^{44}$, ils sont stéréotypés comme les nôtres, mais la nageoire caudale est une gerbe de cinq ou de six tiges, alors que celle des nôtres ressemble à un calice de lleur bifide, tout comme sur la mosaïque Flacheron ${ }^{45}$. Cette forme de la nageoire

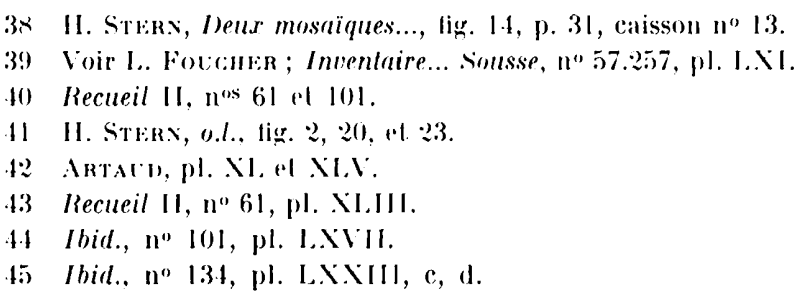

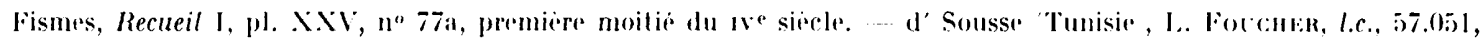

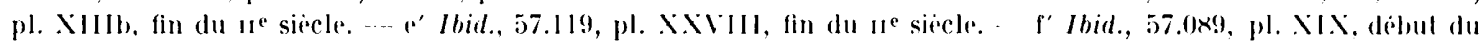

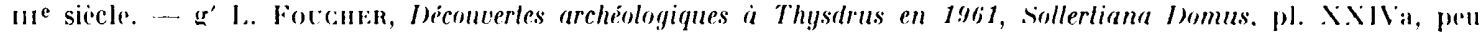

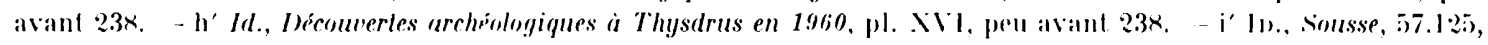
pl. XXXIth, milien du me siecle.

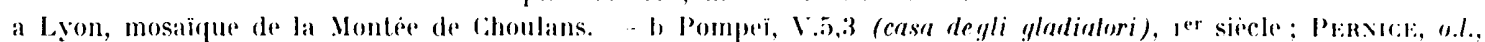

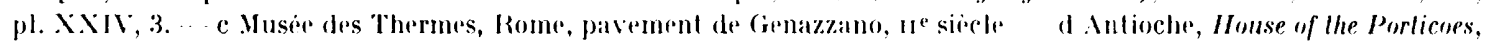

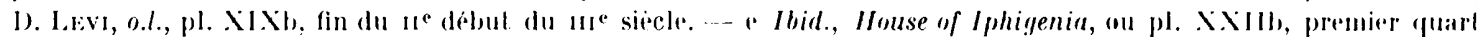

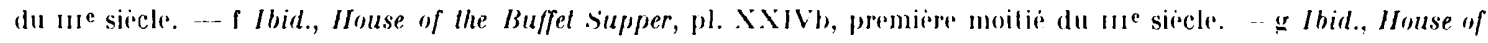
Dionysos and Ariadne, pl. XXIXc, premier quart du ni ${ }^{\mathrm{e}}$ siecle. - h Ihid.. IIouse of the Boal of I'syches. pl. XXXVIt, milieu du ne siecle. 
caudale se trouve ailleurs en Gaule ${ }^{46}$ et en Suisse où elle prend parfois l'aspect d'une véritable plante trifide ${ }^{47}$. C'est une stylisation trìs ancienne qui remonte à l'art grec d'où elle a passé dans l'art italique et dans celui des provinces.

Par la composition et les motifs de remplissage, la mosaïque de la Vontée de Choulans est donc une création typique de l'école rhodanienne et plus particulièrement des ateliers de Lyon. Ie qualité moyenne, elle ne dépasse pas le nivealu de la production courante. L'utilisation de cubes de verre (canards) trahit cependant un soin dans l'exécution qui n'est pas fréquent. Le type classique de la couronne de lauriers et la sobriété du décor (quadrillage de simples filets) invitent à la placer tout au début du groupe des ouvres qui marquent l'essor de cette école sous les Sévères. En l'état acturl de nos connaissances, et étant donné l'absence de tout indice archéologique extéricur, je la daterais très approximativement de l'époque antonine.

Henri STERN.

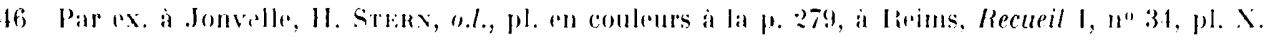

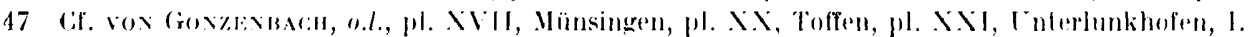

